\title{
Vascular Permeability: Regulation Pathways and Role in Kidney Diseases
}

\author{
Anxiang Cai Christos Chatziantoniou Amélie Calmont \\ Unité mixte Inserm - Sorbonne Université, UMR_S1155, Tenon Hospital, Paris, France
}

\section{Keywords}

Vascular permeability - Vascular endothelial cadherin . Acute kidney injury · Chronic kidney disease · Diabetic kidney disease

\begin{abstract}
Background: Vascular permeability (VP) is a fundamental aspect of vascular biology. A growing number of studies have revealed that many signalling pathways govern VP in both physiological and pathophysiological conditions. Furthermore, emerging evidence identifies VP alteration as a pivotal pathogenic factor in acute kidney injury, chronic kidney disease, diabetic kidney disease, and other proteinuric diseases. Therefore, perceiving the connections between these pathways and the aetiology of kidney disease is an important task as such knowledge may trigger the development of novel therapeutic or preventive medical approaches. In this regard, the discussion summarizing VP-regulating pathways and associating them with kidney diseases is highly warranted. Summary: Major pathways of VP regulation comprise angiogenic factors including vascular endothelial growth factor/VEGFR, angiopoietin/Tie, and class 3 semaphorin/neuropilin and inflammatory factors including hista-
\end{abstract}

karger@karger.com

(c) 2021 S. Karger AG, Basel

www.karger.com/nef

Karger' mine, platelet-activating factor, and leukocyte extravasation. These pathways mainly act on vascular endothelial cadherin to modulate adherens junctions of endothelial cells (ECs), thereby augmenting VP via the paracellular pathway. Elevated VP in diverse kidney diseases involves EC apoptosis, imbalanced regulatory factors, and many other pathophysiological events, which in turn exacerbates renal structural and functional disorders. Measures improving VP effectively ameliorate the diseased kidney in terms of tissue injury, endothelial dysfunction, kidney function, and long-term prognosis. Key Messages: (1) Angiogenic factors, inflammatory factors, and adhesion molecules represent major pathways that regulate VP. (2) Vascular hyperpermeability links various pathophysiological processes and plays detrimental roles in multiple kidney diseases.

(c) 2021 S. Karger AG, Basel

\section{Introduction}

For vertebrates, the vascular system plays indispensable roles in storing blood, nourishing tissues with oxygen and nutrients, transporting metabolites, and offering gateways to the immune system. To accomplish these 


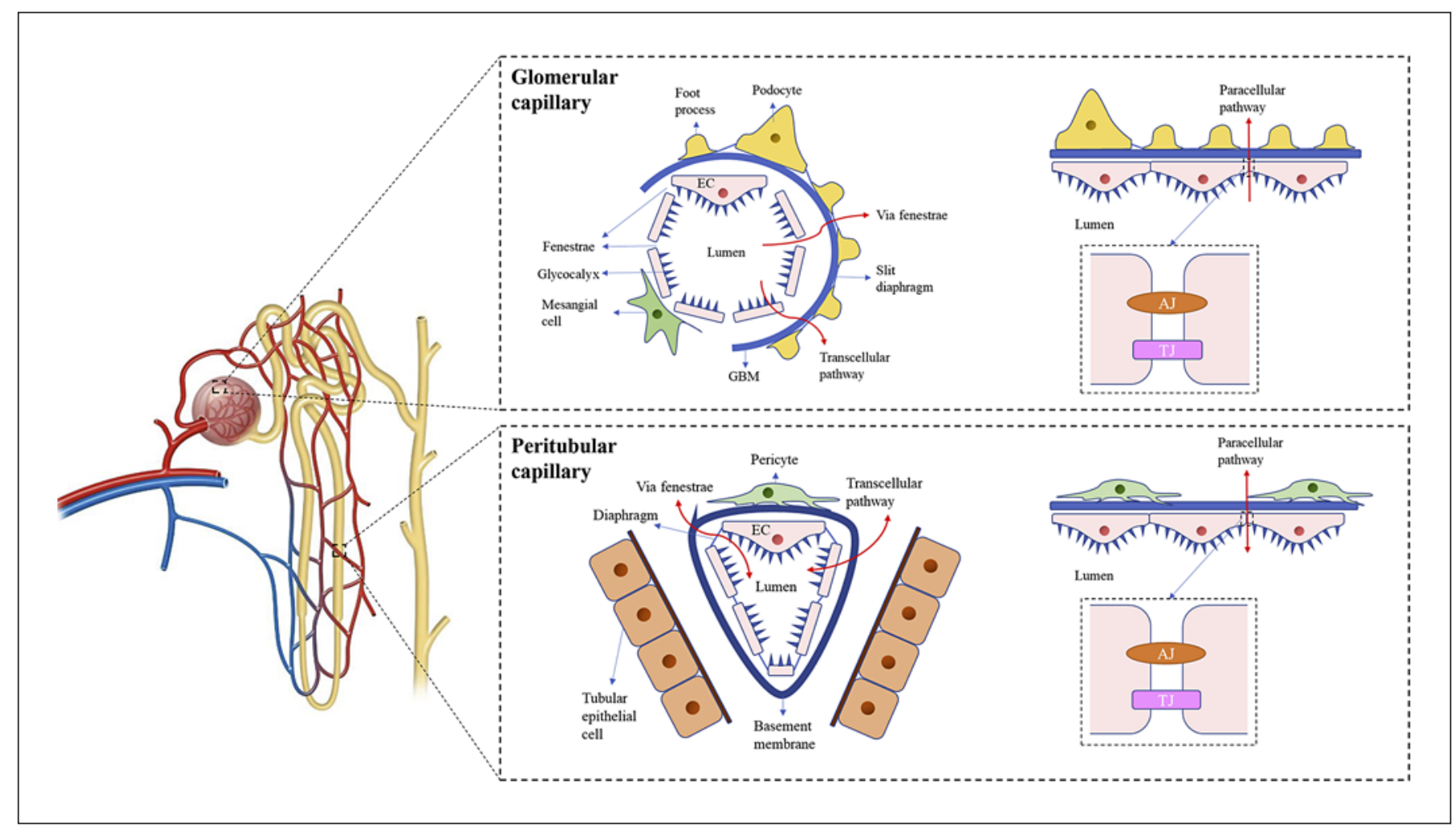

Fig. 1. Renal capillary beds and permeability pathways. Schematic summary of the elements and pathways of VP in renal capillaries. The kidney has 2 types of capillary beds, the glomerular capillary and the post-glomerular capillary (peritubular capillary), which have different elements that impact their permeability. Both of them comprise endothelial surface glycocalyx and EC monolayer for the transcellular pathway, EC junctions (TJs and AJs) for the paracellular pathway, EC fenestrae (peritubular capillary fenestrae has a thin diaphragm), pericytes (mesangial cells as glomerular pericytes), and basement membrane (GBM). Furthermore, the glomerular permeability barrier includes the podocytes with the slit diaphragm between their foot processes. VP, vascular permeability; EC, endothelial cell; TJs, tight junctions; AJs, adherens junctions; GBM, glomerular basement membrane.

missions, it provides adequate interfaces and sufficient permeability for the material exchange between the circulation and different tissues [1].

Vascular permeability (VP) is generally defined as blood vessels' ability to control the bidirectional passage of molecules and immune cells with a certain range of size and to restrict the extravasation of larger molecules. Under physiological conditions, molecules $<40 \mathrm{kDa}$ can pass through mature vessels, whereas larger proteins, such as albumin $(66 \mathrm{kDa})$ and transferrin $(80 \mathrm{kDa})$, are retained. However, under pathophysiological conditions, for instance, inflammation and allergy, even molecules of 2,000 $\mathrm{kDa}$ may extravasate. In addition to size and physiological status, VP is also affected by the type of microvessels involved (venules or capillaries), other characteristics of the molecule (shape, charge, and hydrophilicity), and the histological pathway (transcellular, paracellular, or via fenestrae) [2].
Kidney vasculature is unique and complex: the renal artery enters the kidney at the renal hilum and further branches into the interlobular and arcuate arteries that eventually form the glomerular capillaries and post-glomerular capillaries. This specific vasculature ensures the kidney's function of blood ultrafiltration, urine production, and the maintenance of the liquid, electrolyte, and acid-base equilibrium [3]. As a vital property of the kidney vasculature, VP of both glomerular and post-glomerular capillaries plays crucial roles in kidney homeostasis. The glomerular permeability of the filtration barrier determines the types of molecules to be filtered from serum to Bowman's capsule. Moreover, VP of the post-glomerular capillaries, including peritubular capillary network and the vasa recta, is essential to the ultra-filtrate reabsorption and the medullary osmotic gradient maintenance [4].

VP alteration is closely related to various kidney diseases. In acute kidney injury (AKI), capillary hyperpermeabil- 
ity contributes to microvascular hypoperfusion, oedema, hypoxia, and inflammation, further aggravating tissue injury and dysfunction [5]. Alterations of systemic and glomerular permeability are associated with albuminuria and oedema in multiple proteinuric diseases such as diabetic kidney diseases (DKD), idiopathic nephrotic syndrome (INS), and hypertension [6-8]. For chronic kidney disease (CKD), particularly end-stage renal disease (ESRD), microvascular leakage accompanied by capillary rarefaction and tubulointerstitial fibrosis were reported both in animals and patients, associated with poor prognosis $[9,10]$.

Considering the pivotal role of VP in kidney homeostasis under both physiological and pathological conditions, further investigation of its regulation mechanism and its relation to kidney diseases is highly warranted. This review will discuss the current research progress of VP regulation by major signalling pathways, VP alteration in various kidney diseases, their correlation, and translation of such molecular mechanisms to preclinical and clinical practice. The elements and pathways of VP in renal capillaries are summarized in Figure 1.

\section{Regulation of VP}

\section{VEGF/VEGFR Signalling Pathway}

Vascular endothelial growth factor (VEGF) was initially named vascular permeability factor, indicating its key roles in permeability regulation. VEGF family members comprise VEGF-A to VEGF-E and placental growth factor, which act via their receptors VEGFR1, VEGFR2, and VEGFR3 and co-receptors neuropilin-1 (NRP1) and neuropilin-2 (NRP2) [11].

VEGF-A/VEGFR2 with the co-receptor NRP1 is the main pathway to modulate angiogenesis and VP [11]. In the kidney, VEGF is produced primarily by podocytes and tubular epithelial cells, and VEGFR2 is expressed on endothelial cells (ECs), where it forms a mechanosensory complex with vascular endothelial cadherin (VE-cadherin), the major adhesive protein at adherens junctions (AJs). VEGF stimulation triggers VEGFR2 phosphorylation at tyrosine $\mathrm{Y} 949$ that binds the T-cell-specific adaptor, further eliciting downstream Src/Vav2/Rac1/PAK1 signal, leading to VE-cadherin phosphorylation at Y658 and Y685 and then endocytosis followed by degradation or recycling, thus augmenting VP $[12,13]$. Another VEGFR2 phosphorylation site responsible for VP is Y1173, which may, through PLC-dependent calcium influx or PI3K/Akt pathway-induced endothelial nitric oxide synthase (eNOS) phosphorylation at serine S1177, ac-

Vascular Permeability and Kidney

Diseases tivate eNOS to produce NO [12]. NO induces vasodilation and local blood flow increase, thus resulting in altered shear stress to modulate VE-cadherin phosphorylation and hydrostatic pressure change to promote intravascular component extravasation $[14,15]$. Besides, NO mediates S-nitrosylation of $\beta$-catenin at Cys619, promoting its dissociation from VE-cadherin and AJs disassembly [16]. Also, the VEGF/VEGFR2 pathway may mediate VP via disrupting tight junctions (TJs) [17].

Interestingly, VEGFR3 with the co-receptor NRP2 was formerly considered to control lymphangiogenesis, but VEGFR3 may also modulate VP via suppressing VEGFR2 expression and VEGF/VEGFR2 pathway activity in quiescent, angiogenic ECs [18]. Such phenomenon implies the interaction between VEGFRs and emphasizes their synthetic effect when focusing on any of these pathways, even though the VEGFRs present discrepant affinity for different ligands. Of note, the data discussed above are generated by using murine models [12-18] and ECs cultured in vitro, including human $[14,15,17]$ and mouse $[14-16,18]$ EC lines, which may not perfectly reflect the signalling transduction process in the human body. Moreover, it should be emphasized that the internalization of VE-cadherin as the common pathway of VEGFinduced VP leads to the disruption of AJs and finally mediates VP via the paracellular pathway.

\section{Angpt/Tie and VE-PTP}

Angiopoietins (Angpts) are secreted growth factors that regulate angiogenesis and inflammation via endothelial receptor tyrosine kinase Tie. Angpt1 and Angpt2 act antagonistically in VP modulation: Angpt1 is constitutively expressed in non-ECs, it phosphorylates Tie 2 on ECs, promotes its redistribution, and further elicits the Rap1/Rac1 pathway to deactivate RhoA/ROCK pathwaymediated phosphorylation of myosin light chain, rearrangement of VE-cadherin, and vascular leakage $[19,20]$. Conversely, Angpt2 expression by ECs is usually controlled at low levels but upregulated under pathological conditions and competitively binds to Tie 2 to inactivate it [20]. Also, Angpt2 decreases claudin-5 expression in TJs, increases caveolin-1 expression to support the transcellular pathway, and stimulates pericyte detachment and migration to mediate VP [21]. Another possible mechanism of Angpt2-induced VP may involve the damage of the endothelial surface glycocalyx that is important to endothelial dysfunction, as a clinical trial suggests that increased Angpt 2 may mediate the association between low-density lipoprotein-cholesterol and glycocalyx injury marked by the biomarker syndecan- 1 in patients with 
nephrotic syndrome [22]. Tie1 is less characterized than Tie2, which might directly interact with Tie2 to regulate the Angpt/Tie2 pathway, and its deletion tightens the AJs by upregulating VE-cadherin expression [23].

Vascular endothelial protein tyrosine phosphatase (VEPTP) is an endothelial-specific phosphatase associated with both VE-cadherin and Tie2, and it stabilizes VE-cadherin in the quiescent endothelium via inhibiting GEF-H1mediated RhoA activation and in the activated endothelium via dephosphorylating VE-cadherin at Y658/Y685 [24]. Controversially, it might play dual roles in the challenged endothelium, where its inhibition activates Tie2 to stabilize AJs. However, in the absence of Tie2, VE-PTP inhibition augments VP, consistent with its effect on VEcadherin dephosphorylation $[19,24]$. Furthermore, VEPTP dephosphorylates VEGFR2 in Ang1/Tie2-dependent manner, which inhibits VE-cadherin phosphorylation [25]. In turn, during VEGF stimulation or leukocyte extravasation, VE-PTP is dissociated from VE-cadherin to open AJs [26]. In conclusion, VE-PTP regulates VP through its combined action on VE-cadherin dephosphorylation, Tie2 suppression, and VEGFR2 regulation. Simultaneous targeting VEGF, Angpt2, and VE-PTP could be a promising therapeutic strategy for microangiopathy [27].

Similar to research studies on VEGF, these data are obtained mainly from mouse models [19-21, 23, 27], human EC lines [19, 21, 23, 27], and mouse EC lines [21, 24-26], demonstrating the effects of the Angpt/Tie signal via the transcellular [21] and paracellular [19, 20, 24, 25] pathways. The clinical trial [22] provides evidence of how Angpt2 modulates VP via glycocalyx damage in patients, and more clinical studies are required to add to the reliability of current conclusions.

\section{Class 3 Semaphorin and NRP1}

Class 3 semaphorins are secreted soluble molecules initially identified as axonal guidance proteins. NRP1 is a membrane-anchored receptor for class 3 semaphorins in vertebrates, which can non-competitively bind VEGF and trigger corresponding downstream pathways [28].

NRP1 as a co-receptor of VEGF/VEGFR2 can add to ligand-receptor affinity and participate in signal transmission [11, 28]. Furthermore, NRP1 and VEGFR2 can either mediate VP independent of each other [29]. For example, the SEMA3A/NRP1 pathway inhibits PP2A activity through the Src/Set signal that leads to VE-cadherin phosphorylation at serine S665 and internalization or triggers the PI3K/Akt pathway [30, 31]. Also, SEMA3A may interact with the VEGFR1/NRP2 complex to elicit Mical2 activation, F-actin disorganization, and cytoskel- eton disruption [32]. Moreover, our investigation identified SEMA3C as a pro-permeable factor that contributes to tissue injury during AKI. Of interest, SEMA3A is antiangiogenic and to some degree antagonizes VEGF-A, whereas it does promote VP [31]; SEMA3C functions are similar to VEGF, as it promotes angiogenesis and VP in mice (Cai A et al., data in revision). These results indicate that the SEMA3A (SEMA3C)/NRP1 pathway may participate in various diseases by modulating VP, which involves not only the pathway itself but also its interaction with VEGFR. As for possible therapy, we may inhibit specifically the class 3 semaphorin binding site on NRP1 to block the permeability response, while VEGF binding left intact to maintain EC proliferation and survival.

Collectively, the source of these data is the combination of both in vivo experiments using murine models [28-32] and in vitro cell culture using human EC lines [29-31] or murine EC lines [32]. It is suggested that class 3 semaphorins binding to NRP1 to induce VP via the paracellular pathway, but how to translate it into clinical practice needs further investigation.

\section{Inflammatory Factors and Leukocyte Extravasation}

Vascular hyperpermeability caused by various inflammatory factors plays critical parts in inflammation and anaphylaxis. Histamine, a prominent inflammatory factor, has 4 cognate $G$ protein-coupled receptors, designated H1R to H4R. Histamine increases VP through H1R, which can also be activated by the platelet-activating factor (PAF). H1R activation promotes PLC-mediated mobilization of calcium and the RhoA/ROCK pathway, which together induce myosin light chain phosphorylation and VE-cadherin redistribution, followed by gap formation [33]. Besides the aforementioned eNOS pathway, involving haemodynamic changes and NO-induced Snitrosylation of $\beta$-catenin, p120 and VE-cadherin, represent another major pro-permeable mechanism of these inflammatory factors $[34,35]$. Additionally, TNF- $\alpha$, another famous pro-inflammatory cytokine, strongly promotes permeability via triggering the RhoA/ROCK signal, activating the Rac/ROS pathway to dissociate VEPTP from VE-cadherin, and modulating TJ molecules, including ZO-1, occludin, and claudin [36, 37].

Leukocyte extravasation, a key event in inflammation, is closely associated with VP through VE-cadherin, since anti-permeability via the VE-cadherin- $\alpha$-catenin complex strongly reduced leukocyte infiltration in certain tissues without affecting VEGFR2 signal, VE-PTP association, or cytoskeletal organization [13, 38]. Intriguingly, VP and leukocyte extravasation are regulated by different 




Fig. 2. Major signalling pathways regulating VP. These pathways mainly involve the angiogenic signalling pathway (VEGF/ VEGFR2, Angpt/Tie2, and SEMA3A/NRP1) and the inflammatory signalling pathway (histamine/H1R, PAF/PAFR, TNF- $\alpha$ / TNFR, and leukocyte/VCAM-1). Regardless of the precise process of signal transduction, they modulate the common substrate, VEcadherin, in the AJ. VE-cadherin phosphorylation at Y658, Y685, and 5665 leads to internalization followed by degradation or recycling, thereby increasing VP. VE-PTP inhibits VE-cadherin and VEGFR2 phosphorylation to stabilize endothelial junctions; therefore, its dissociation from VE-cadherin increases VP. S-nitrosyl-

tyrosines of VE-cadherin, as phosphorylation at Y685 enables VP and leukocyte dephosphorylates Y731 to extravasate [39]. Notwithstanding VE-PTP functions selectively on Y685, it is critical in leukocyte transmigration because VEGF stimulation or lymphocyte binding to vascular cell adhesion molecule-1 activates the Rac1/ NOX/ROS/Pyk2 pathway that mediates VE-PTP detachment from VE-cadherin, required for VP and leukocyte diapedesis in vivo [26]. Furthermore, VE-cadherin Y685 phosphorylation supports chemokine diffusion from the inflamed interstitium to vascular lumen, thereby promoting the reverse trans-endothelial migration of activated neutrophils to induce remote organ damage [40].

These preclinical research studies on inflammatory cytokines and cells are all based on murine models [33-40], ation of VE-cadherin, $\beta$-catenin, and p120 mediates the disruption of AJs and VE-cadherin endocytosis. The phosphorylation of MLC, the stress fibre, promotes VE-cadherin rearrangement and redistribution to open the endothelial contact. Leukocyte dephosphorylates VE-cadherin Y731 to induce extravasation. VP is also modulated by TJs, caveolin-1 expression (transcellular transportation), pericyte detachment, and haemodynamic factors. VP, vascular permeability; VE-cadherin, vascular endothelial cadherin; AJ, adherens junction; VE-PTP, vascular endothelial protein tyrosine phosphatase; MLC, myosin light chain; TJs, tight junctions; IS, indoxyl sulphate; EC, endothelial cell. with the help of human EC lines [33, 35, 38, 39] or isolated primary cells $[36,39]$. Taking together, these inflammatory factors share several common mechanisms amid not only themselves but also angiogenic factors, and modification of VE-cadherin to facilitate the paracellular pathway seems to play central roles. The pathways regulating VP are summarized in Figure 2.

\section{VP in Kidney Diseases}

\section{EC Apoptosis in Kidney Diseases}

The capillary endothelium consists of an EC monolayer, whose massive death disrupts endothelial integrity and increases VP. Extreme physical, chemical, and bio- 




Fig. 3. Factors leading to EC apoptosis in kidney diseases. The chemotherapeutic drug cisplatin results in EC apoptosis at low concentration. In ischaemic AKI, hypovolaemia causes hypoxia and haemodynamic changes, which promotes ROS generation to induce EC apoptosis. During CKD, the uraemic toxin IS stimulates monocytes to produce TNF- $\alpha$, thereby promoting cytotoxic T-cell recruitment and EC apoptosis. Dyslipidaemia contributes to EC apoptosis via inhibiting the PI3K/PDK1/Akt/mTOR pathway and the PI3K/Akt/eNOS pathway. Hyperglycaemia elicits the ROS/Akt/NF- $\kappa \mathrm{B}$ pathway to mediate EC apoptosis. These 2 metabolic disorders synergize to induce endothelial damage in DKD. EC, endothelial cell; IS, indoxyl sulphate; TJ, tight junction; AJ, adherens junction.

logical stimuli may lead to unregulated EC death, named necrosis. Nevertheless, recent studies focus more on EC apoptosis, a form of programmed cell death inducible by various factors including nephrotoxin, haemodynamic change, and metabolic disorders, which represent, respectively, major pathogenic factors in AKI, CKD, and DKD. The widely used chemotherapeutic drug cisplatin as a nephrotoxin induces EC apoptosis at low concentrations and necrosis at high concentrations, thus contributing to AKI in both patients and animal models [41]. Indoxyl sulphate, a typical uraemic toxin in CKD, stimulates monocytes to express TNF- $\alpha$ that promotes ECs to recruit $\mathrm{CD} 4{ }^{+} \mathrm{CD} 28^{-} \mathrm{T}$ cells via CX3CL1/CX3CR1 bind- ing, thereby provoking endothelial damage in ESRD patients [42]. Moreover, during hypovolemic shock, the hypoxia and haemodynamic changes support the generation of reactive oxygen species (ROS), which induces the apoptosis of human umbilical vein endothelial cells (HUVECs) and may contribute to pre-renal AKI [43]. Of note, there may be crosstalk between dyslipidaemia and hyperglycaemia, in which protein kinase B (also called Akt) activation plays a central role. In high-fat diet-induced mouse aortic EC and human aortic EC apoptosis, PI3K/ $\mathrm{PDK} 1 / \mathrm{Akt} / \mathrm{mTOR}$ pathway activation is inhibited, whereas activation of the PI3K/Akt/eNOS pathway attenuates inflammation and endothelial dysfunction in mouse 
aortic ECs induced by oxidized low-density lipoprotein, suggesting protective effects of Akt and downstream pathways in dyslipidaemia [44, 45]. Conversely, activation of the ROS/Akt/NF- $\kappa$ B pathway acts detrimentally in hyperglycaemia-induced HUVEC apoptosis [46]. Dyslipidaemia is identified as an independent risk factor of $\mathrm{DKD}$, but the mechanism linking dyslipidaemia and hyperglycaemia remains largely unclear. Further investigation should elucidate their underlying relationship during EC apoptosis, which would contribute to the prevention and treatment of $\mathrm{DKD}$ and other vascular complications. The factors leading to EC apoptosis in kidney diseases are summarized in Figure 3.

\section{$V P$ and $A K I$}

AKI is defined as a sharp loss of kidney excretory function, characterized by rapid declined glomerular filtration rate, increased plasma nitrogenous wastes, and variably diminished urinary output. Tubular epithelial cell perturbation contributes greatly to AKI, whereas emerging evidence has unveiled the essential role of endothelial dysfunction [5].

Capillary hyperpermeability in AKI involves multiple factors. First, the endothelial monolayer is directly disrupted during $\mathrm{AKI}$, as there exists a relative late-phase EC apoptosis in ischaemic AKI mice [47], and the modification of endothelial barrier molecules such as glycocalyx and VE-cadherin occurs in post-cardiac surgery AKI pigs [48]; septic AKI also contributes to EC apoptosis via endotoxin and overactivated immune system [5]. Moreover, in ischaemic AKI murine models, pericytes show an increased expression of matrix metalloproteinases (MMPs, a family of zinc-dependent endopeptidases that degrade various proteins in the extracellular matrix), especially MMP7 and MMP9, while pericytes downregulate the tissue inhibitor of metalloproteinase 3 to degrade the extracellular matrix and promotes av $\beta 5$ integrin-induced pericyte detachment and migration $[49,50]$. Third, infiltrated neutrophils in the interstitium release cytokines, chemokines, proteases, and ROS to activate the complement system, upregulate inflammatory factors, and induce adhesion molecules, thereby aggravating tissue damage and endothelial dysfunction in ischaemic AKI mice [51]. Furthermore, while both tubuloglomerular feedback and imbalanced vasomotor factors contribute to renal vasoconstriction [5], the consequent altered haemodynamic force exacerbates VP [15]. These effects involve EC apoptosis [5, 47], endothelial surface glycocalyx [48], cell-cell contacts and paracellular pathway [15, 48, $51]$, pericytes, and extracellular matrix $[49,50]$. They syn- chronize to disrupt vascular integrity and result in microvascular hypoperfusion, interstitial oedema, hypoxia, and increased tubular pressure and tubular obstruction, finally contributing to glomerular filtration rate decrease and tissue injury [5].

VP-regulating molecules alter significantly in AKI. During sepsis, VE-cadherin shedding and plasma soluble VE-cadherin levels are associated with severe AKI and organ dysfunctions in sepsis patients, implying endothelial AJ disruption and leukocyte reverse trans-endothelial migration [40, 52]. Besides, the disbalance of Angpts, including decreased Angpt1 and elevated Angpt2, is associated with AKI in critically ill patients independently of inflammation [53]. Especially, although the VEGF/ VEGFR2 pathway mediates VP, it might play beneficial roles in AKI. Higher levels of plasma VEGF and placental growth factor are associated with lower risks for AKI and mortality in post-cardiac surgery patients, whereas higher VEGFR1 levels with higher risks, probably due to the effect of VEGF on EC survival and angiogenesis [54]. Moreover, our studies identified SEMA3C as a pro-angiogenic and pro-permeable factor that acts deleterious roles in ischaemic and nephrotoxic AKI mouse models through aggravating VP (Cai A et al., data in revision).

Undoubtedly, vascular integrity restoration treatment alleviates AKI. Adenosine 2A agonists can reduce neutrophil infiltration to inhibit renal VP and preserve renal function in ischaemic AKI mice [51]. Erythropoietin (EPO) may attenuate septic AKI in mice via enhancing the EPO/EPOR and VEGF/VEGFR2 signal and inhibiting HIF-1 $\alpha$, iNOS, and NF- $\kappa B$ expressions, linking tissue hypoxia, endothelial dysfunction, and inflammation [55]. Furthermore, adhesion molecules and pericytes act pivotal parts, as inhibiting pericyte activation and stabilizing microvasculature through blocking $\alpha v \beta 5$ integrin can attenuate VP and protect against ischaemic AKI in mice [50]. However, activated pericytes and unresolved VP may render the kidney revascularization strategy futile, and failed kidney recovery leads to AKI to CKD transition [56].

\section{$V P$ and $C K D$}

CKD is defined as abnormalities of kidney structure or function of at least 3-month duration with health implications, which severely burdens public health. Irrespective of the aetiology, peritubular capillaries undergo similar ultra-structural and functional changes during progressive renal disease in both mice and humans, including capillary rarefaction and microvascular hyperpermeability [9]. 
Causes of vascular hyperpermeability in CKD differ from those in AKI while sharing some common features. First, a rat model shows that endothelial surface layer glycocalyx encounters a similar loss in CKD, associating proteinuria with systemic endothelial dysfunction [57]. Second, studies using patients' biopsies and mouse models have unravelled that fibroblasts in renal fibrosis principally derive from pericytes, whose activation is both pro-permeable and pro-fibrotic, linking AKI to CKD [56]. Also, mouse models indicate that pericyte deficiency and imbalanced VEGF expression impact both existing vessels and neovascularization, resulting in leaky and immature new vessels $[11,58]$. Besides, specific rheological changes at microvascular branches make them major sites of vascular leakage in both AKI and CKD mouse models (Cai A et al., data in revision) [9]. Moreover, a study using HUVECs suggests that chronic inflammation in CKD triggers the activation of NF- $\mathrm{KB}$ and downstream pro-inflammatory factors in CKD and ESRD patients [59]. Moreover, serum uraemic toxins of CKD patients elicit VE-cadherin disruption and F-actin reorganization, thus opening up AJs in HUVECs [60]. The toxins may also come from normal proteins, as HDL becomes noxious particles in CKD patients which inhibit endothelial NO production, destabilize the endothelial barrier, and promote vascular dysfunction of human aortic ECs [61]. In turn, vascular hyperpermeability contributes to CKD progression. Glomerular endothelial leakage results in abnormal passage of plasma protein into the renal tubules and interstitium to elicit the inflammatory and fibrotic response, associated with systemic vascular dysfunction and cardiovascular complications in CKD patients [62]. Extravasated serum proteins such as fibrinogen may also disrupt TJs of rat cardiac microvascular ECs [9]. As for the permeability elements, VP in CKD involves glycocalyx [57], pericytes [56], EC junctions, and paracellular pathways $[9,59-61]$. As a result, the disrupt endothelial barrier participates in haemodynamic changes including hypoperfusion, haemoconcentration, and interstitial oedema, which promote hypoxia, cell swelling, inflammation, and platelet activation within the kidney, leading to augmented interstitial pro-fibrotic cytokines and impaired cell functions of degrading collagen. Altogether, these pathophysiological processes contribute to protein malnutrition, body fluid overload, renal fibrogenesis, and worse survival in CKD patients $[9,10]$.

It is worth emphasizing that vascular hyperpermeability belongs to endothelial dysfunction, an important endothelial alteration in $\mathrm{CKD}$, characterized by impaired NO bioavailability [63]. NO is generated from L-arginine via eNOS catalysis, which not only regulates VP but also shows vasorelaxant, anti-inflammatory, and anti-thrombotic properties. In CKD patients, eNOS is inhibited by multiple pathophysiological events, such as oxidative stress (OS), inflammation, hyperphosphataemia, dyslipidaemia, and endogenous inhibitor of eNOS, including asymmetric dimethylarginine and symmetric dimethylarginine $[63,64]$. In consequence, the disordered endothelium presents abnormal activation, reduced proliferation and elevated apoptosis, and disrupt endothelial barrier function $[63,64]$. These alterations contribute to renal fibrosis and systemic atherosclerosis, associated with poor clinical outcomes [63].

Among the major components of endothelial dysfunction, OS represents a pivotal point correlated with VP. In OS, oxidative molecules, most commonly ROS, overwhelm antioxidant defence mechanisms [65]. Such a status is present even in the early stages of CKD, caused by chronic inflammation, mitochondrial dysfunction, and excessive ROS generation [65]. During CKD progression, OS exacerbates along with reduced kidney function and severely burdens ESRD patients requiring haemodialysis (HD) and peritoneal dialysis (PD) [66, 67]. As aforementioned, ROS participates in inflammatory factor-induced VP and EC apoptosis to destabilize both renal and systemic vascular integrity, contributing to body overhydration; OS can also promote leukocyte recruitment, inhibit eNOS, and suppress angiogenesis, thereby aggravating both local and systemic endothelial dysfunction, renal fibrogenesis, and atherogenesis in CKD and especially in ESRD patients in need of dialysis [65]. The key event of OS is the formation of oxidative products, which is impacted by lifestyle and diet habits, and by different factors in HD and PD: HD-related factors include dialyser membranes, anticoagulant usage, dialysate, medication administration, and HD duration [66]. In contrast, OS status in PD mainly depends on the composition of the PD solution, in which high glucose and acidic $\mathrm{pH}$ are considered as the culprit. Rodent models and clinical studies have revealed that such PD fluids promote the generation of the advanced glycation end-products and ROS to induce OS and break redox homeostasis, thereby contributing to increased systemic and local inflammation, peritoneal cell apoptosis, and peritoneal fibrosis [67-69]. In consequence, the microvascular density of the peritoneum is decreased while the microvessels undergo hyperpermeability, resulting in loss of peritoneal integrity and its transport function $[67,68]$. As for treatment, antioxidant supplementation may represent possible therapeutic strategies $[67,68]$; neutral-pH, low-GDP PD fluids can 
Table 1. Clinical-related studies of VP in CKD

\begin{tabular}{|c|c|c|c|}
\hline Study [ref.] & Year & Methodology & Results \\
\hline Serradell et al. [70] & 2002 & $\begin{array}{l}\text { Patient serum: } \\
10 \text { HD } \\
\text { Paired controls } \\
\text { HUVECs }\end{array}$ & $\begin{array}{l}\text { Endothelial dysfunction and inflammation marked by expression, } \\
\text { redistribution, and shedding of adhesion molecules: } \\
\text { HD > control }\end{array}$ \\
\hline Yu et al. [10] & 2012 & $\begin{array}{l}\text { Patients: } \\
41 \mathrm{PD}\end{array}$ & $\begin{array}{l}\text { (1) Systemic albumin leak measured by trans-capillary escape rate of albumin: } \\
\text { PD > non-uraemic } \\
\text { (2) Complex relationship between endothelial barrier, inflammation, and } \\
\text { hypoalbuminaemia }\end{array}$ \\
\hline Caballo et al. [58] & 2012 & $\begin{array}{l}\text { Patient serum: } \\
15 \text { controls } \\
15 \text { CKD } \\
15 \text { HD and } 9 \text { PD } \\
\text { HUVECs }\end{array}$ & $\begin{array}{l}\text { Endothelial activation and damage marked by activation of p } 38 \text { MAPK and } \\
\text { NF- } \kappa B \text { in HUVECs: } \\
\mathrm{PD}>\mathrm{HD} \approx \text { pre-dialysis }>\text { control }\end{array}$ \\
\hline Shroff et al. [60] & 2014 & $\begin{array}{l}\text { Child patient serum: } \\
12 \text { controls } \\
39 \text { CKD (stage 2-5) } \\
20 \text { dialysis } \\
23 \text { transplants } \\
\text { HAECs }\end{array}$ & $\begin{array}{l}\text { Endothelial dysfunction marked by (1) NO production } \downarrow \text {, superoxide production } \\
\uparrow, \text { and VCAM- } 1 \text { expression } \uparrow \text { in CKD-HDL-treated cells; (2) Urate, Angpt2, } \\
\text { IL-6, and SDMA } \uparrow \text { in patient serum: dialysis > CKD } 4-5>\text { CKD } 2-3>\text { control, } \\
\text { partial recovery in transplant group }\end{array}$ \\
\hline Seliger et al. [61] & 2016 & $\begin{array}{l}\text { Older hypertensive } \\
\text { patients: } \\
30 \text { non-CKD } \\
36 \text { CKD (stage } 1-4) \\
\text { (11 albuminuria) }\end{array}$ & $\begin{array}{l}\text { Microvascular endothelial function marked by microvascular reactivity: } \\
\text { albuminuria }<\text { normoalbuminuria } \\
\text { CKD }<\text { non-CKD }\end{array}$ \\
\hline Tsai et al. [71] & 2017 & $\begin{array}{l}\text { Patients: } \\
290 \text { CKD (stage 3-5) }\end{array}$ & $\begin{array}{l}\text { (1) Angpt } 2 \text { positively, significantly correlated with fluid overload } \\
\text { (2) Risks for commencing dialysis and rapid renal function decline: fluid } \\
\text { overload, high circulating Angpt } 2>\text { low overhydration, low circulating Angpt } 2\end{array}$ \\
\hline Schaefer et al. [69] & 2018 & $\begin{array}{l}\text { Child patient } \\
\text { peritoneal and } \\
\text { omental specimens: } \\
56 \text { controls } \\
90 \text { ESRD } \\
82 \text { PD } \\
\text { Follow-up biopsies: } \\
24 \text { PD (13 months) }\end{array}$ & $\begin{array}{l}\text { Neutral-pH and low-glucose degradation product dialysis fluids induce } \\
\text { peritoneal inflammation, fibroblast activation, EMT, and angiogenesis to impact } \\
\text { peritoneal permeability and transport function }\end{array}$ \\
\hline $\begin{array}{l}\text { Vila Cuenca et al. } \\
\text { [59] }\end{array}$ & 2019 & $\begin{array}{l}\text { Patient plasma: } \\
6 \text { non-CKD } \\
6 \text { CKD } \\
\text { HUVECs }\end{array}$ & $\begin{array}{l}\text { (1) Endothelial barrier function marked by trans-endothelial electrical } \\
\text { resistance: } \\
\text { CKD < non-CKD } \\
\text { (2) Disrupt cell-cell junctions in CKD group } \\
\text { (3) Rescuable by VD supplementation }\end{array}$ \\
\hline
\end{tabular}

HD, haemodialysis; HUVECs, human umbilical vein endothelial cells; PD, peritoneal dialysis; MAPK, mitogen-activated protein kinase; NF- $\mathrm{B}$, nuclear factor kappa-B; HAECs, human aortic endothelial cells; VCAM-1, vascular cell adhesion molecule-1; HDL, highdensity lipoprotein; Angpt2, angiopoietin 2; IL-6, interleukin-6; SDMA, symmetric dimethylarginine; EMT, epithelial-mesenchymal transition; VD, vitamin D.

Vascular Permeability and Kidney Diseases 
induce early inflammation, epithelial-mesenchymal transition, and angiogenesis of the peritoneum to impact its transport function in child patients, but their long-term effect requires further investigation [70].

Similar to AKI, there exists severe dysregulation of permeability regulatory factors in CKD. Reduced VEGF levels in different renal fibrosis rodent models might account for capillary rarefaction and loss of EC fenestrations [9]. Nevertheless, VP was still elevated with more formation of caveolae and cytoplasmic vesicles in HUVECs treated with uraemic patient serum, implying the involvement of other factors such as imbalanced Angpts [71]. Indeed, Angpt2-mediated VP aggravates fluid overload, facilitates inflammatory response, and implies adverse renal outcomes in CKD patients [72]. Moreover, VE-cadherin, opposite to its shedding in AKI, is upregulated in CKD mice but might be disrupted by uraemic toxins $[60,73]$.

Considering its important roles in $\mathrm{CKD}$, targeting VP represents potential therapeutics. Vitamin D supplementation enforces HUVEC integrity via strengthening VEcadherin, stabilizing cortical F-actin ring formation, and reducing MMP9, thereby attenuating endothelial dysfunction in CKD [60]. Overexpression of vasoprotective VEGF- $A_{165}$ b isoform protects against glomerular hyperpermeability, ultra-structural abnormalities, and proteinuria in CKD mouse [58]. Targeting the impaired Angpt/ Tie pathway, Angpt1 administration preserves pericyte coverage and enhances EC junctions to attenuate peritoneal VP and inflammation, thus ameliorating peritoneal transport function in $\mathrm{PD}$ rats [74]. To improve peritoneal permeability and transport function, antioxidants such as $\mathrm{N}$-acetylcysteine can protect against OS and dipeptide alanyl-glutamine may activate pathways associated with the protection of the PM integrity in mice [6769]. The clinical-related studies of VP in CKD are summarized in Table 1.

\section{$V P$ and $D K D$}

DKD refers to chronic renal dysfunction in patients with diabetes, belonging to diabetic microvascular complications. Albeit enormous efforts taken to control diabetes, DKD remains the leading cause of ESRD [6].

Diabetes-mediated glomerular leakage is closely linked to metabolic disorders. First, hyperglycaemia can induce HUVEC apoptosis probably via ROS production [46]. Second, studies using mouse models and human renal glomerular EC (GEC) lines reported that hyperglycaemia activates the protein kinase $\mathrm{C}$ (PKC) system to facilitate GEC apoptosis and contributes to renal matrix produc- tion $[75,76]$. Third, elevated circulating platelet microparticles in DKD impair ROS and NO system and cause GEC injury in rats and isolated primary rat GECs [77]. Furthermore, dyslipidaemia as an independent risk factor of diabetes participates in endothelial dysfunction. Both patient biopsies and rodent models showed that lipids may stimulate TGF- $\beta$ to induce ROS production and GEC damage, while triglyceride-rich lipoproteins can degrade glycocalyx [78]. The mechanisms of VP in DKD are mainly caused by the toxicity of hyperglycaemia and dyslipidaemia, involving GEC apoptosis [46, 75-78] and glycocalyx degradation [78]. Resultantly, endothelial hyperpermeability impairs filtration barrier function and contributes to albuminuria, the key event of DKD and subsequent complications [6].

As a supplement to classic diabetes therapy, treatment targeting aberrant angiogenic factors levels to inhibit VP has broad prospects. First, proper VEGF-A levels are necessary for renal microvasculature maintenance in diabetes, whereas excessive VEGF-A contributes to glomerular leakage accompanied by nodular glomerulosclerosis and massive proteinuria. More precisely, vasoactive isoform VEGF- $\mathrm{A}_{165} \mathrm{a}$ is the culprit, whereas VEGF- $\mathrm{A}_{165}$ b normalizes glomerular permeability of both human and rodents via VEGFR2 phosphorylation and glycocalyx restoration in GECs, whose upregulation implies well-preserved kidney function [58]. Besides, using mouse models and human GEC lines, researchers found that VEGF-C counteracts VEGF-A effect via preventing VEGFR alteration and maintaining endothelial glycocalyx to protect diabetic glomerulus and inhibit DKD development [79]. Furthermore, the C-peptide may inhibit VEGF-induced ROS generation, stress fibre formation, and VE-cadherin disassembly, thereby preventing VEGF-mediated VP and improving renal microvascular flow in diabetic mice [80]. Second, the Angpt/Tie system also participates, as Angpt 1 levels decrease in diabetes accompanied by albuminuria and renal morphologic alterations. Its glomerular repletion in diabetic mice significantly reduces albuminuria and GEC proliferation, probably through increased Tie2 phosphorylation, elevated soluble VEGFR1 levels, decreased VEGFR2 phosphorylation, and increased eNOS activity [81]. Moreover, VE-PTP is robustly upregulated in diabetic mice renal microvasculature, whose inhibition enhances Tie 2 activity, activates eNOS, and reduces the expression of pro-inflammatory and profibrotic genes, thus decreasing VP and inflammation to preserve microvasculature and kidney function in $\mathrm{DKD}$ [82]. 


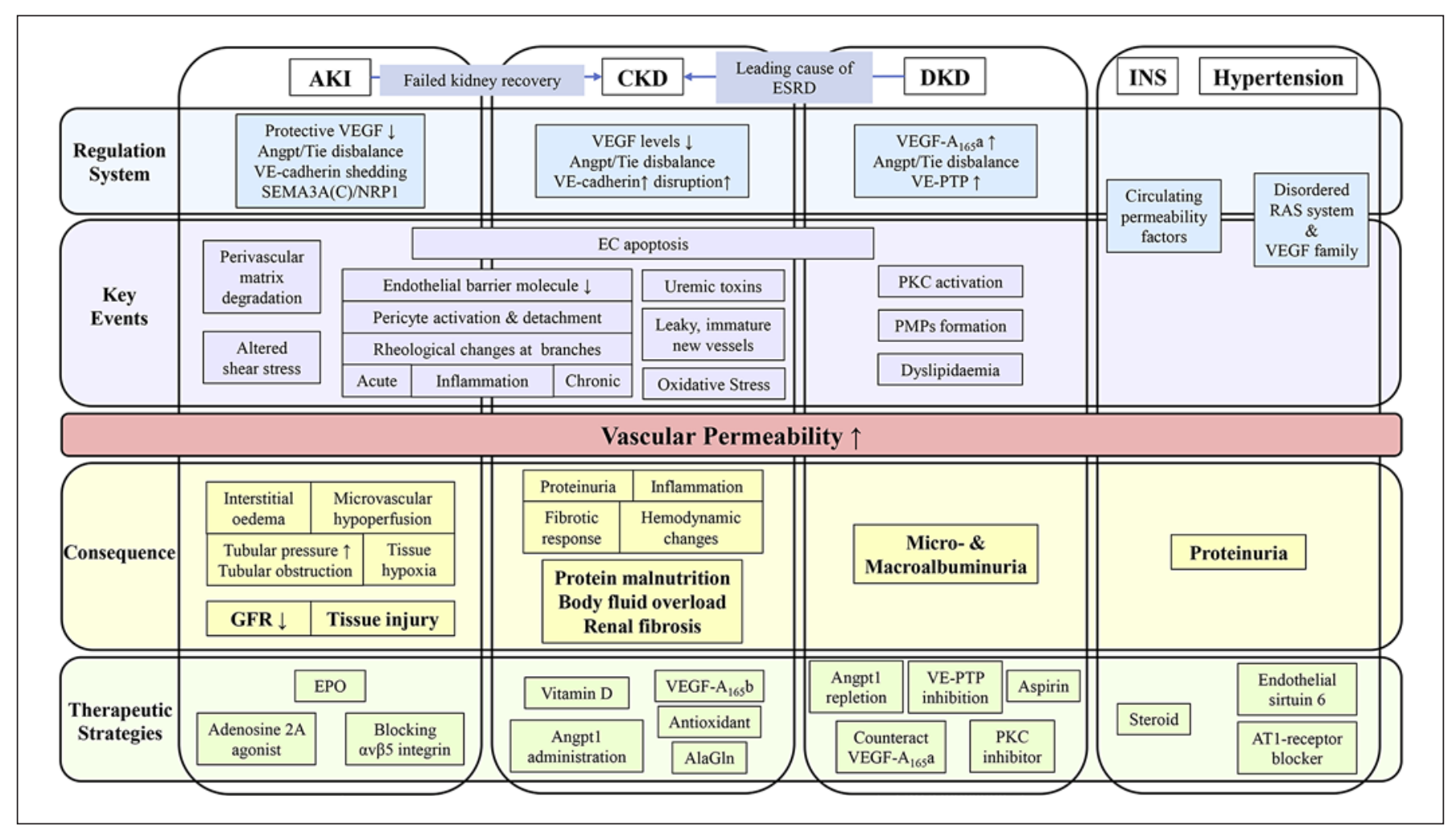

Fig. 4. VP in kidney diseases. Schematic summary linking permeability regulation and kidney diseases. Briefly, in different kidney diseases, disordered regulatory molecules participate in the key pathophysiological events that increase permeability. Consequently, vascular hyperpermeability further contributes to the development and progression of these diseases. Treatments targeting the regulation system and key events may represent promising therapeutic strategies for these kidney diseases. VP, vascular permeability.

Other aetiologies of vascular hyperpermeability may also provide possible targets. The PKC- $\beta$ inhibitor LY333531 attenuates GEC apoptosis, while the PKC- $\alpha$ and PKC- $\beta$-dual inhibitor CGP41252 prevents albuminuria development and reduces existing albuminuria in diabetic mice $[75,76]$. Aspirin can reduce platelet microparticle formation to ameliorate GEC injury, albuminuria, glomerular hypertrophy, and mesangial matrix expansion in early DKD mice without affecting blood glucose levels [77].

\section{Other Kidney Diseases}

INS, characterized by massive proteinuria, hypoalbuminaemia, and oedema, mainly consists of minimal change disease and focal segmental glomerulosclerosis. In INS, researchers have identified, via utilizing patient specimens and murine models, multiple circulating permeability factors, including haemopexin in minimal change disease and soluble urokinase receptor, cardiotrophin-like cytokine factor 1, apolipoprotein A-1, calcium/ calmodulin-serine protein kinase, and CD40 agonist in focal segmental glomerulosclerosis [7]. These factors target GEC surface glycocalyx, glomerular basement membrane, and podocytes to disrupt the integrity of the glomerular filtration barrier [7]. In consequence, abnormal glomerular leakage results in proteinuria, while capillary hyperpermeability contributes to oedema formation which might be significantly reduced by steroid treatment [83].

Hypertension remains a leading cause of morbidity and mortality worldwide, with hypertensive nephropathy being its interdependent part. The disordered renin-angiotensin system plays key roles in hypertension, whose inhibition effectively delays and reduces albuminuria. Independent of haemodynamic effects, angiotensin II acts on the podocyte AT1-receptor to increase nephrin- $\beta$ arrestin2 binding and nephrin endocytosis, thus augmenting glomerular permeability and contributing to albuminuria in mice; therefore, AT1-receptor blockers may prevent albuminuria even in normotensives [8]. More- 
over, endothelial sirtuin 6 can ameliorate angiotensin IIinduced VP and increase vascular NO bioavailability to alleviate endothelial dysfunction in hypertensive mice, thereby protecting against hypertension and associated cardiorenal injury [84]. Furthermore, clinical trials have revealed that inhibition of the VEGF/VEGFR2 signal often accompanies proteinuria and hypertension, which probably involves the eNOS pathway [85]. The association between hypertension and dysregulated VEGF family complicates this promising chemotherapeutic strategy and requires further investigations. The linkage between VP and kidney diseases is summarized in Figure 4.

\section{Conclusive Remarks}

Over the past decades, increasing clinical and preclinical investigations have unravelled the deleterious roles of vascular hyperpermeability in various kidney diseases. Extensive research has identified major pathways regulating VP, which provides potential biomarkers to predict the severity and prognosis of diseases such as AKI and CKD. Furthermore, targeting these pathways may have broad prospects for the intervention of renal diseases.
However, despite encouraging results obtained from mouse models, clinical trials focusing on VP in kidney disease remain rare, probably owing to a lack of practical methods to target specifically VP. To translate these findings into clinical practice, future investigations should further test the efficacity of such biomarkers and develop credible tools to normalize VP in kidney diseases.

\section{Conflict of Interest Statement}

The authors have no conflicts of interest to declare.

\section{Funding Sources}

This study was supported by an Academy de Medicine fellowships to A. Cai, and a Marie Curie Personal Fellowship [704450, Horizon2020] to A. Calmont.

\section{Author Contributions}

A. Calmont and A. Cai designed the study. A. Cai wrote the manuscript and prepared the figures with inputs from all the authors.

\section{References}

1 Filipowska J, Tomaszewski KA, Niedźwiedzki Ł, Walocha JA, Niedźwiedzki T. The role of vasculature in bone development, regeneration and proper systemic functioning. Angiogenesis. 2017;20(3):291-302.

2 Egawa G, Nakamizo S, Natsuaki Y, Doi H, Miyachi Y, Kabashima K. Intravital analysis of vascular permeability in mice using twophoton microscopy. Sci Rep. 2013;3:1932.

3 McMahon AP. Development of the mammalian kidney. Curr Top Dev Biol. 2016;117:31-64.

4 Barry DM, McMillan EA, Kunar B, Lis R, Zhang T, Lu T, et al. Molecular determinants of nephron vascular specialization in the kidney. Nat Commun. 2019;10(1):5705.

5 Basile DP, Anderson MD, Sutton TA. Pathophysiology of acute kidney injury. Compr Physiol. 2012;2(2):1303-53.

6 Molitch ME, Adler AI, Flyvbjerg A, Nelson RG, So WY, Wanner C, et al. Diabetic kidney disease: a clinical update from kidney disease: improving global outcomes. Kidney Int. 2015; 87(1):20-30.

7 Candelier JJ, Lorenzo HK. Idiopathic nephrotic syndrome and serum permeability factors: a molecular jigsaw puzzle. Cell Tissue Res. 2020;379(2):231-43.

8 Königshausen E, Zierhut UM, Ruetze M, Potthoff SA, Stegbauer J, Woznowski M, et al. Angiotensin II increases glomerular permea- bility by $\beta$-arrestin mediated nephrin endocytosis. Sci Rep. 2016;6:39513.

9 Babickova J, Klinkhammer BM, Buhl EM, Djudjaj S, Hoss M, Heymann F, et al. Regardless of etiology, progressive renal disease causes ultrastructural and functional alterations of peritubular capillaries. Kidney Int. 2017;91(1):70-85.

10 Yu Z, Tan BK, Dainty S, Mattey DL, Davies SJ. Hypoalbuminaemia, systemic albumin leak and endothelial dysfunction in peritoneal dialysis patients. Nephrol Dial Transplant. 2012;27(12):4437-45.

11 Ferrara N, Adamis AP. Ten years of anti-vascular endothelial growth factor therapy. Nat Rev Drug Discov. 2016;15(6):385-403.

12 Koch S, Claesson-Welsh L. Signal transduction by vascular endothelial growth factor receptors. Cold Spring Harb Perspect Med. 2012;2(7):a006502.

13 Giannotta M, Trani M, Dejana E. VE-cadherin and endothelial adherens junctions: active guardians of vascular integrity. Dev Cell. 2013;26(5):441-54.

14 Di Lorenzo A, Lin MI, Murata T, Landskroner-Eiger S, Schleicher M, Kothiya M, et al. eNOS-derived nitric oxide regulates endothelial barrier function through VE-cadherin and Rho GTPases. J Cell Sci. 2013;126(Pt 24): 5541-52.
15 Orsenigo F, Giampietro C, Ferrari A, Corada M, Galaup A, Sigismund S, et al. Phosphorylation of VE-cadherin is modulated by haemodynamic forces and contributes to the regulation of vascular permeability in vivo. Nat Commun. 2012;3:1208.

16 Thibeault S, Rautureau Y, Oubaha M, Faubert D, Wilkes BC, Delisle C, et al. S-nitrosylation of beta-catenin by eNOS-derived NO promotes VEGF-induced endothelial cell permeability. Mol Cell. 2010;39(3):468-76.

17 Li R, Qi Y, Jiang M, Zhang T, Wang H, Wang $\mathrm{L}$, et al. Primary tumor-secreted VEGF induces vascular hyperpermeability in premetastatic lung via the occludin phosphorylation/ ubiquitination pathway. Mol Carcinog. 2019; 58(12):2316-26.

18 Heinolainen K, Karaman S, D'Amico G, Tammela T, Sormunen R, Eklund L, et al. VEGFR3 modulates vascular permeability by controlling VEGF/VEGFR2 signaling. Circ Res. 2017;120(9):1414-25.

19 Frye M, Dierkes M, Küppers V, Vockel M, Tomm J, Zeuschner D, et al. Interfering with VE-PTP stabilizes endothelial junctions in vivo via Tie-2 in the absence of VE-cadherin. J Exp Med. 2015;212(13):2267-87.

20 Eklund L, Kangas J, Saharinen P. AngiopoietinTie signalling in the cardiovascular and lymphatic systems. Clin Sci. 2017;131(1):87-103. 
21 Gurnik S, Devraj K, Macas J, Yamaji M, Starke J, Scholz A, et al. Angiopoietin-2-induced blood-brain barrier compromise and increased stroke size are rescued by VE-PTPdependent restoration of Tie2 signaling. Acta Neuropathol. 2016;131(5):753-73.

22 Chaves MMS, Mendes MS, Schwermann MP, Queiroz R, Coelho RF, Salmito FTS, et al. Angiopoietin-2: a potential mediator of the glycocalyx injury in adult nephrotic patients. J Clin Med. 2018;7(11):401.

23 La Porta S, Roth L, Singhal M, Mogler C, Spegg C, Schieb B, et al. Endothelial Tie1-mediated angiogenesis and vascular abnormalization promote tumor progression and metastasis. J Clin Invest. 2018;128(2):834-45.

24 Juettner VV, Kruse K, Dan A, Vu VH, Khan Y, Le J, et al. VE-PTP stabilizes VE-cadherin junctions and the endothelial barrier via a phosphatase-independent mechanism. J Cell Biol. 2019;218(5):1725-42.

25 Hayashi M, Majumdar A, Li X, Adler J, Sun Z, Vertuani S, et al. VE-PTP regulates VEGFR2 activity in stalk cells to establish endothelial cell polarity and lumen formation. Nat Commun. 2013;4:1672.

26 Vockel M, Vestweber D. How T cells trigger the dissociation of the endothelial receptor phosphatase VE-PTP from VE-cadherin. Blood. 2013;122(14):2512-22.

27 Shen J, Frye M, Lee BL, Reinardy JL, McClung JM, Ding K, et al. Targeting VE-PTP activates TIE2 and stabilizes the ocular vasculature. J Clin Invest. 2014;124(10):4564-76.

28 Gelfand MV, Hagan N, Tata A, Oh WJ, Lacoste B, Kang KT, et al. Neuropilin-1 functions as a VEGFR2 co-receptor to guide developmental angiogenesis independent of ligand binding. Elife. 2014;3:e03720.

29 Fantin A, Lampropoulou A, Senatore V, Brash JT, Prahst C, Lange CA, et al. VEGF165induced vascular permeability requires NRP1 for ABL-mediated SRC family kinase activation. J Exp Med. 2017;214(4):1049-64.

30 Le Guelte A, Galan-Moya EM, Dwyer J, Treps L, Kettler G, Hebda JK, et al. Semaphorin 3A elevates endothelial cell permeability through PP2A inactivation. J Cell Sci. 2012;125(Pt 17): 4137-46.

31 Acevedo LM, Barillas S, Weis SM, Göthert JR, Cheresh DA. Semaphorin 3A suppresses VEGF-mediated angiogenesis yet acts as a vascular permeability factor. Blood. 2008; 111(5):2674-80.

32 Hou ST, Nilchi L, Li X, Gangaraju S, Jiang SX, Aylsworth A, et al. Semaphorin3A elevates vascular permeability and contributes to cerebral ischemia-induced brain damage. Sci Rep. 2015;5:7890.

33 Mikelis CM, Simaan M, Ando K, Fukuhara S, Sakurai A, Amornphimoltham P, et al. RhoA and ROCK mediate histamine-induced vascular leakage and anaphylactic shock. Nat Commun. 2015;6:6725

34 Nakamura T, Murata T. Regulation of vascular permeability in anaphylaxis. Br J Pharmacol. 2018;175(13):2538-42.
35 Guequén A, Carrasco R, Zamorano P, Re bolledo L, Burboa P, Sarmiento J, et al. S-nitrosylation regulates VE-cadherin phosphorylation and internalization in microvascular permeability. Am J Physiol Heart Circ Physiol. 2016;310(8):H1039-44.

36 Fang M, Zhong WH, Song WL, Deng YY, Yang DM, Xiong B, et al. Ulinastatin ameliorates pulmonary capillary endothelial permeability induced by sepsis through protection of tight junctions via inhibition of TNF- $\alpha$ and related pathways. Front Pharmacol. 2018;9: 823.

37 Marcos-Ramiro B, García-Weber D, Millán J. TNF-induced endothelial barrier disruption: beyond actin and Rho. Thromb Haemost. 2014;112(6):1088-102.

38 Schulte D, Küppers V, Dartsch N, Broermann A, Li H, Zarbock A, et al. Stabilizing the VEcadherin-catenin complex blocks leukocyte extravasation and vascular permeability. Embo J. 2011;30(20):4157-70.

39 Wessel F, Winderlich M, Holm M, Frye M, Rivera-Galdos R, Vockel M, et al. Leukocyte extravasation and vascular permeability are each controlled in vivo by different tyrosine residues of VE-cadherin. Nat Immunol. 2014; 15(3):223-30

40 Owen-Woods C, Joulia R, Barkaway A, Rolas L, Ma B, Nottebaum AF, et al. Local microvascular leakage promotes trafficking of activated neutrophils to remote organs. J Clin Invest. 2020;130(5):2301-18.

41 Ozkok A, Edelstein CL. Pathophysiology of cisplatin-induced acute kidney injury. Biomed Res Int. 2014;2014:967826.

42 Kim HY, Yoo TH, Hwang Y, Lee GH, Kim B, Jang J, et al. Indoxyl sulfate (IS)-mediated immune dysfunction provokes endothelial damage in patients with end-stage renal disease (ESRD). Sci Rep. 2017;7(1):3057.

43 Chao Y, Ye P, Zhu L, Kong X, Qu X, Zhang J, et al. Low shear stress induces endothelial reactive oxygen species via the AT1R/eNOS/ NO pathway. J Cell Physiol. 2018;233(2): 1384-95.

44 Li Y, Yang C, Zhang L, Yang P. MicroRNA-210 induces endothelial cell apoptosis by directly targeting PDK1 in the setting of atherosclerosis. Cell Mol Biol Lett. 2017;22:3.

45 Chen L, Qin L, Liu X, Meng X. CTRP3 alleviates $\mathrm{Ox}$-LDL-induced inflammatory response and endothelial dysfunction in mouse aortic endothelial cells by activating the PI3K/Akt/ eNOS pathway. Inflammation. 2019;42(4): $1350-9$.

46 Guan G, Han H, Yang Y, Jin Y, Wang X, Liu $X$. Neferine prevented hyperglycemia-induced endothelial cell apoptosis through suppressing ROS/Akt/NF- $\mathrm{kB}$ signal. Endocrine. 2014;47(3):764-71.

47 Yang B, Lan S, Dieudé M, Sabo-Vatasescu JP, Karakeussian-Rimbaud A, Turgeon J, et al. Caspase- 3 is a pivotal regulator of microvascular rarefaction and renal fibrosis after ischemia-reperfusion injury. J Am Soc Nephrol. 2018;29(7):1900-16.
48 Qureshi SH, Patel NN, Murphy GJ. Vascular endothelial cell changes in postcardiac surgery acute kidney injury. Am J Physiol Renal Physiol. 2018;314(5):F726-f35.

49 Schrimpf C, Xin C, Campanholle G, Gill SE, Stallcup W, Lin SL, et al. Pericyte TIMP3 and ADAMTS1 modulate vascular stability after kidney injury. J Am Soc Nephrol. 2012;23(5): 868-83.

50 McCurley A, Alimperti S, Campos-Bilderback SB, Sandoval RM, Calvino JE, Reynolds TL, et al. Inhibition of $\alpha v \beta 5$ integrin attenuates vascular permeability and protects against renal ischemia-reperfusion injury. J Am Soc Nephrol. 2017;28(6):174152.

51 Bolisetty S, Agarwal A. Neutrophils in acute kidney injury: not neutral any more. Kidney Int. 2009;75(7):674-6.

52 Yu WK, McNeil JB, Wickersham NE, Shaver CM, Bastarache JA, Ware LB. Vascular endothelial cadherin shedding is more severe in sepsis patients with severe acute kidney injury. Crit Care. 2019;23(1):18.

53 Robinson-Cohen C, Katz R, Price BL, HarjuBaker S, Mikacenic C, Himmelfarb J, et al. Association of markers of endothelial dysregulation Ang1 and Ang2 with acute kidney injury in critically ill patients. Crit Care. 2016;20(1): 207.

54 Mansour SG, Zhang WR, Moledina DG, Coca SG, Jia Y, Thiessen-Philbrook H, et al. The association of angiogenesis markers with acute kidney injury and mortality after cardiac surgery. Am J Kidney Dis. 2019;74(1): 36-46.

55 Stoyanoff TR, Rodríguez JP, Todaro JS, Colavita JPM, Torres AM, Aguirre MV. Erythropoietin attenuates LPS-induced microvascular damage in a murine model of septic acute kidney injury. Biomed Pharmacother. 2018; 107:1046-55

56 Venkatachalam MA, Weinberg JM, Kriz W, Bidani AK. Failed tubule recovery, AKI-CKD transition, and kidney disease progression. J Am Soc Nephrol. 2015;26(8):1765-76.

57 Salmon AH, Ferguson JK, Burford JL, Gevorgyan $\mathrm{H}$, Nakano D, Harper SJ, et al. Loss of the endothelial glycocalyx links albuminuria and vascular dysfunction. J Am Soc Nephrol. 2012;23(8):1339-50.

58 Kikuchi R, Stevens M, Harada K, Oltean S, Murohara T. Anti-angiogenic isoform of vascular endothelial growth factor-A in cardiovascular and renal disease. Adv Clin Chem. 2019;88:1-33.

59 Caballo C, Palomo M, Cases A, Galán AM, Molina P, Vera M, et al. NFkB in the development of endothelial activation and damage in uremia: an in vitro approach. PLoS One. 2012; 7(8):e43374.

60 Vila Cuenca M, van Bezu J, Beelen RHJ, Vervloet MG, Hordijk PL. Stabilization of cell-cell junctions by active vitamin $\mathrm{D}$ ameliorates uraemia-induced loss of human endothelial barrier function. Nephrol Dial Transplant. 2019;34(2):252-64. 
61 Shroff R, Speer T, Colin S, Charakida M, Zewinger S, Staels B, et al. HDL in children with CKD promotes endothelial dysfunction and an abnormal vascular phenotype. J Am Soc Nephrol. 2014;25(11):2658-68.

62 Seliger SL, Salimi S, Pierre V, Giffuni J, Katzel L, Parsa A. Microvascular endothelial dysfunction is associated with albuminuria and CKD in older adults. BMC Nephrol. 2016; 17(1):82.

63 Roumeliotis S, Mallamaci F, Zoccali C. Endothelial dysfunction in chronic kidney disease, from biology to clinical outcomes: a 2020 update. J Clin Med. 2020;9(8):2359.

64 Jamwal S, Sharma S. Vascular endothelium dysfunction: a conservative target in metabolic disorders. Inflamm Res. 2018;67(5):391405.

65 Scioli MG, Storti G, D’Amico F, Rodriguez Guzman R, Centofanti F, Doldo E, et al. Oxidative stress and new pathogenetic mechanisms in endothelial dysfunction: potential diagnostic biomarkers and therapeutic targets. J Clin Med. 2020;9(6):1995.

66 Liakopoulos V, Roumeliotis S, Gorny X, Dounousi E, Mertens PR. Oxidative stress in hemodialysis patients: a review of the literature. Oxid Med Cell Longev. 2017;2017:3081856.

67 Liakopoulos V, Roumeliotis S, Gorny X, Eleftheriadis T, Mertens PR. Oxidative stress in patients undergoing peritoneal dialysis: a current review of the literature. Oxid Med Cell Longev. 2017;2017:3494867.

68 Roumeliotis S, Dounousi E, Salmas M, Eleftheriadis T, Liakopoulos V. Unfavorable effects of peritoneal dialysis solutions on the peritoneal membrane: the role of oxidative stress. Biomolecules. 2020;10(5):768.

69 Boehm M, Herzog R, Klinglmüller F, Lichtenauer AM, Wagner A, Unterwurzacher M, et al. The peritoneal surface proteome in a model of chronic peritoneal dialysis reveals mechanisms of membrane damage and preservation. Front Physiol. 2019;10:472.
70 Schaefer B, Bartosova M, Macher-Goeppinger S, Sallay P, Vörös P, Ranchin B, et al. Neutral $\mathrm{pH}$ and low-glucose degradation product dialysis fluids induce major early alterations of the peritoneal membrane in children on peritoneal dialysis. Kidney Int. 2018;94(2): 419-29.

71 Serradell M, Díaz-Ricart M, Cases A, Zurbano MJ, López-Pedret J, Arranz O, et al. Uremic medium causes expression, redistribution and shedding of adhesion molecules in cultured endothelial cells. Haematologica. 2002; 87(10):1053-61.

72 Tsai YC, Chiu YW, Kuo HT, Lee JJ, Lee SC, Chen TH, et al. The interaction between fluid status and angiopoietin-2 in adverse renal outcomes of chronic kidney disease. PLoS One. 2017;12(3): $\mathrm{e} 0173906$

73 Yamaguchi I, Tchao BN, Burger ML, Yamada M, Hyodo T, Giampietro C, et al. Vascular endothelial cadherin modulates renal interstitial fibrosis. Nephron Exp Nephrol. 2012;120(1): e20-31.

74 Shi Y, Xiong Y, Lei Y, Li Z, Yan H, Yuan J, et al. Protective effect of COMP-angiopoietin-1 on peritoneal vascular permeability and peritoneal transport function in uremic peritoneal dialysis rats. Am J Transl Res. 2019;11(9): 5932-43.

75 Menne J, Shushakova N, Bartels J, Kiyan Y, Laudeley R, Haller H, et al. Dual inhibition of classical protein kinase $\mathrm{C}-\alpha$ and protein kinase $C-\beta$ isoforms protects against experimental murine diabetic nephropathy. Diabetes. 2013;62(4):1167-74.

76 Wang ZB, Zhang S, Li Y, Wang RM, Tong LC, Wang Y, et al. LY3333531, a PKC $\beta$ inhibitor, attenuates glomerular endothelial cell apoptosis in the early stage of mouse diabetic nephropathy via down-regulating swiprosin-1. Acta Pharmacol Sin. 2017;38(7):1009-23.
77 Zhang Y, Ma KL, Gong YX, Wang GH, Hu ZB, Liu L, et al. Platelet microparticles mediate glomerular endothelial injury in early diabetic nephropathy. J Am Soc Nephrol. 2018; 29(11):2671-95.

78 Rutledge JC, Ng KF, Aung HH, Wilson DW. Role of triglyceride-rich lipoproteins in diabetic nephropathy. Nat Rev Nephrol. 2010; 6(6):361-70.

79 Onions KL, Gamez M, Buckner NR, Baker SL, Betteridge KB, Desideri S, et al. VEGFC reduces glomerular albumin permeability and protects against alterations in VEGF receptor expression in diabetic nephropathy. Diabetes. 2019;68(1):172-87.

80 Lim YC, Bhatt MP, Kwon MH, Park D, Lee S, Choe J, et al. Prevention of VEGF-mediated microvascular permeability by C-peptide in diabetic mice. Cardiovasc Res. 2014;101(1): 155-64.

81 Dessapt-Baradez C, Woolf AS, White KE, Pan J, Huang JL, Hayward AA, et al. Targeted glomerular angiopoietin-1 therapy for early diabetic kidney disease. J Am Soc Nephrol. 2014; 25(1):33-42.

82 Carota IA, Kenig-Kozlovsky Y, Onay T, Scott $\mathrm{R}$, Thomson BR, Souma T, et al. Targeting VE-PTP phosphatase protects the kidney from diabetic injury. J Exp Med. 2019;216(4): 936-49.

83 Siddall EC, Radhakrishnan J. The pathophysiology of edema formation in the nephrotic syndrome. Kidney Int. 2012;82(6):635-42.

84 Guo J, Wang Z, Wu J, Liu M, Li M, Sun Y, et al. Endothelial SIRT6 is vital to prevent hypertension and associated cardiorenal injury through targeting Nkx3.2-GATA5 signaling. Circ Res. 2019;124(10):1448-61.

85 Estrada CC, Maldonado A, Mallipattu SK. Therapeutic inhibition of VEGF signaling and associated nephrotoxicities. J Am Soc Nephrol. 2019;30(2):187-200. 